\title{
Ecole Pratique des Hautes Etudes (VIe Section): la recherche en Afrique Noire
}

UN enseignement d'initiation à la recherche africaniste fonctionnera au cours de l'année universitaire I 962-3 à l'École Pratique des Hautes Études, section des sciences économiques et sociales. Le programme d'étude s'étendra du I9 novembre I962 au I er juin 1963 . Il comprendra des cours de préparation à la recherche, des travaux pratiques, des séminaires consacrés à des études de cas, organisés dans chacune des disciplines africanistes suivantes : géographie humaine (M. G. Sautter); histoire (M. H. Brunschwig); langues africaines (MM. P. Alexandre et P.-F. Lacroix); civilisations négro-africaines (M. J. J. Maquet); ethnologie générale (M. P. Mercier); ethnologie politique (M. G. Balandier); ethnologie économique; sociologie et politique économiques (M. J. Boyon); en outre un cours d'anglais sera obligatoire pour tous les étudiants qui ne justifieront pas d'une connaissance suffisante de cette langue.

\section{Institute of African Studies at the University College, Ibadan}

AN Institute of African Studies has been established at the University College, Ibadan, with the assistance of a five-year grant from the Rockefeller Foundation. Three research chairs will be associated with the Institute provided for by a grant from the Ford Foundation. The Director is Dr. Kenneth O. Dike, Principal of the University College, Ibadan, and the Administrative Secretary is Mr. Michael Crowder.

\section{'Die sakralen Häuptlinge der Gurunsi' (Africa, July 1962, pp. 292-3)}

Dans le compte rendu de M. Cornevin sur Die sakralen Häuptlinge der Gurunsi se montre une méprise. Mon étude a expressément le seul but de ne décrire que les faits, fournis par mes recherches sur le terrain et limités au sujet. Des comparaisons avec les populations voisines seront faites dans les monographies ainsi que dans l'étude sur les chefferies africaines à paraître, comme je l'ai expliqué et annoncé dans l'épilogue de mon livre. Par cette raison ne figurent dans sa bibliographie que les travaux cités dans le texte comme apportant des informations importantes au sujet propre. Les titres cités par le critique me sont naturellement connus.

\section{(Communication du Dr. Kunz Dittmer)}

\section{Cornevin a répondu :}

'Le professeur Dittmer s'est légitimement ému de certaines remarques de mon compte rendu de son ouvrage sur Die sakralen Häuptlinge der Gurunsi, notamment celles concernant des lacunes bibliographiques.

'Étant donné l'usage constant qui veut que soit citée la totalité de la bibliographie lorsqu'il s'agit d'une ethnie aussi peu étudiée que les Gourounsi, je n'avais pas compris qu'il s'agissait seulement d'un choix.

'Que le Dr. Dittmer veuille bien accepter ici mes excuses et qu'il me permette de souhaiter non seulement la prochaine publication de l'ouvrage qu'il announce, mais encore la traduction de ses deux livres en français pour que le lecteur voltaique puisse bénéficier de ses études aussi riches que documentées.' 